\title{
Aplicação do indicador de salubridade ambiental (ISA) no município de Frederico Westphalen - RS
}

\section{Application of the environmental health indicator (EHI) in Frederico Westphalen - RS}

Data de entrada: 07/08/2018

- Data de aprovação: $19 / 11 / 2018$

Laura Zachi' | Aline Ferrao Custodio Passini' | Jessica de Oliveira Demarco ${ }^{1 * *}$ | Jéssica Stefanello Cadore ${ }^{1}$

DOI: https://doi.org/10.36659/dae.2020.048

\section{ORCID ID}

Zachi L (D) https://orcid.org/0000-0002-5268-8893

Passini AFC (D) https://orcid.org/0000-0002-8468-490X
Demarco JO (iD https://orcid.org/0000-0001-5255-1873

Cadore JS (DD https://orcid.org/0000-0002-5868-4762

\section{Resumo}

O ISA foi desenvolvido para ser utilizado como instrumento de planejamento de políticas públicas, auxiliando a tomada de decisão em relação aos serviços de saneamento, como abastecimento de água, esgoto sanitário, resíduos sólidos, drenagem urbana e controle de vetores, e em consequência aumentar a salubridade do município, garantindo assim, melhor qualidade de vida da população. Este trabalho teve como objetivo indicar o estado de salubridade ambiental da área urbana de Frederico Westphalen por meio de adaptações na metodologia do ISA/CS, sendo denominado de ISA/FW. O ISA/FW é o indicador de $1^{\text {a }}$ ordem, tendo em sua composição os indicadores de $2^{\mathrm{a}}$ e $3^{\mathrm{a}}$ ordem para realização dos cálculos. A área urbana de FW foi considerada como sendo de média salubridade, demostrando que o município ainda carece de alguns serviços.

Palavras-chave: Salubridade ambiental. Saneamento Básico. Qualidade de Vida. Gestão Pública.

\section{Abstract}

The EHI was developed to be used as a planning tool for public policies, helping the decision making in relation to sanitation services, such as water supply, waste water, solid waste, urban drainage and vector control, and consequently increase the health of the city, thus ensuring better quality of life. This study aimed to indicate the state of the environmental health in the urban area of Frederico Westphalen, through adaptations in the EHI/CS methodology, being denominated EHI/FW. The EHI/FW is the first order indicator, having in its composition the indicators of the second and third order to carry out the calculations. The urban area was considered of medium healthiness, demonstrating that the municipality still lacks some services.

Keywords: Environmental Health. Basic Sanitation. Quality of Life. Public Management.

\footnotetext{
1 Universidade Federal de Santa Maria - Santa Maria - Rio Grande do Sul - Brasil.

* Autora correspondente: jessica-demarcoahotmail.com.
} 


\section{INTRODUÇÃO}

Os serviços de abastecimento de água e saneamento são um dos pilares das sociedades em funcionamento (TAKALA, 2017). No entanto, 2,4 bilhões de pessoas ainda não tinham acesso a melhores condições de saneamento no mundo em 2015 (OMS), causando contaminação ambiental e doenças epidêmicas. Embora a diferença tenha diminuído significativamente desde os anos 1990, com um aumento de $54 \%$ em 1990 para $68 \%$ em 2015 , o desafio permanece impressionante. Atingir a meta de acesso universal ao saneamento requer investimentos tremendos nas próximas décadas (HUTTON; CHASE, 2017; PERARD, 2018).

No ano de 2000, o Brasil possuía aproximadamente 9,9 milhões de domicílios sem acesso ao abastecimento de água por rede geral. No caso do esgoto, pouco menos de vinte milhões de domicílios não possuíam coleta. Há, portanto, um sério déficit de acesso domiciliar aos serviços de saneamento básico no país (SAIANI; TONETO júNIOR, 2010).

Os serviços de saneamento básico são cruciais para proteger as pessoas contra inúmeros problemas de saúde. A melhora nesses serviços provou ser eficaz no controle e eliminação de algumas doenças de veiculação hídrica em muitos países (MONTGOMERY et al., 2018). Segundo Heller (1998), as ações de abastecimento de água e de esgotamento sanitário proporcionam benefícios gerais sobre a saúde da população, tanto direta como indiretamente, resultantes primordialmente do desenvolvimento da localidade atendida.

A Lei 11.145, de 5 de janeiro de 2007, estabelece diretrizes nacionais para o saneamento básico, definido-o como um conjunto de serviços, infraestruturas e instalações operacionais de abastecimento de água, esgotamento sanitário, limpeza urbana, manejo dos resíduos sólidos, drenagem e manejo das águas pluviais urbanas
(BRASIL, 2007). O fornecimento de serviços básicos adequados, como eletricidade, água potável e saneamento, é uma questão de subsistência, sustentabilidade e política pública cada vez mais importante (GAZZEH; ABUBAKAR, 2018).

O Indicador de Salubridade Ambiental (ISA), desenvolvido pela Câmara Técnica do Conselho Estadual de Saneamento de São Paulo (CONESAN) em 1999, envolve a caracterização qualitativa e quantitativa dos serviços de abastecimento de água, esgoto sanitário, limpeza pública, drenagem urbana e controle de vetores, sendo um indicador socioeconômico que objetiva delinear ações compatíveis com as realidades regionais e locais. $O$ objetivo geral do ISA é mensurar de maneira uniforme as condições de saneamento de cada município e determinar suas causas (CONESAN, 1999).

Dessa forma, o presente trabalho tem como objetivo apresentar o estado de salubridade ambiental do Município de Frederico Westphalen do estado do Rio Grande do Sul, por meio da adaptação e aplicação da metodologia do Indicador de Salubridade Ambiental de Cocal do Sul (ISA/ CS). As principais necessidades do município e os pontos satisfatórios serão apontados por meio dos indicadores primários e secundários referentes aos serviços de abastecimento de água, coleta e tratamento de esgotos sanitários, manejo de resíduos sólidos, drenagem e controle de vetores.

\section{METODOLOGIA}

O Indicador de Salubridade Ambiental (ISA) (indicador de $1^{\mathrm{a}}$ ordem) criado pelo CONESAN é calculado pela média ponderada de indicadores de $2^{\mathrm{a}}$ ordem específicos por meio da Eq. 1 e envolve diferentes indicadores de $2^{\mathrm{a}}$ e $3^{\mathrm{a}}$ ordem (CONESAN, 1999).

$$
\begin{aligned}
& \text { ISA }=0,25 \mathrm{I}_{\mathrm{AB}}+0,25 \mathrm{I}_{\mathrm{ES}}+0,25 \mathrm{I}_{\mathrm{RS}}+ \\
& 0,10 \mathrm{I}_{\mathrm{CV}}+0,10 \mathrm{I}_{\mathrm{RH}}+0,05 \mathrm{I}_{\mathrm{SE}}
\end{aligned}
$$


Onde:

$\mathrm{I}_{\mathrm{AB}}=$ Indicador de Abastecimento de Água;

$\mathrm{I}_{\mathrm{ES}}=$ Indicador de Esgoto Sanitário;

$\mathrm{I}_{\mathrm{RS}}=$ Indicador de Resíduos Sólidos;

$\mathrm{I}_{\mathrm{CV}}=$ Indicador de Controle de Vetores;

$\mathrm{I}_{\mathrm{RH}}=$ Indicador de Recursos Hídricos;

$\mathrm{I}_{\mathrm{SE}}=$ Indicador Socioeconômico.

Várias adaptações do ISA de São Paulo foram feitas conforme a necessidade de cada município. Um exemplo é o Indicador de Salubridade Ambiental de João Pessoa (ISA/JP), o qual foi desenvolvido por Batista e Silva (2006), incorporandose $o$ indicador de $2^{\mathrm{a}}$ ordem de drenagem urbana $\left(\mathrm{ID}_{\mathrm{u}}\right)$, dentro do formato apropriado para o índice, demonstrado na Eq. 2.

$\mathrm{I}_{\mathrm{SA}}=0,25 * \mathrm{I}_{\mathrm{AB}}+0,20 * \mathrm{I}_{\mathrm{ES}}+0,20$

$* \mathrm{I}_{\mathrm{ES}}+0,20 * \mathrm{I}_{\mathrm{RS}}+0,10 * \mathrm{I}_{\mathrm{CV}}+0,10$

$* \mathrm{I}_{\mathrm{RH}}+0,10 * \mathrm{I}_{\mathrm{DU}}+0,05 * \mathrm{I}_{\mathrm{SE}}$

A partir do ISA/JP, Baggio (2013) adaptou o ISA para o município de Cocal do Sul - SC, chamado de ISA/CS, fazendo as mudanças necessárias em função da disponibilidade de dados referentes ao ISA/JP e à realidade do município. A proposta para o cálculo do valor do ISA/CS é dada pela média ponderada de indicadores específicos, denominados secundários, através da equação 3 :

$$
\begin{aligned}
& \mathrm{ISA}=0,25 * \mathrm{I}_{\mathrm{AB}}+0,25 * \mathrm{I}_{\mathrm{ES}}+0,20 * \mathrm{I}_{\mathrm{RS}} \\
& +0,20 * \mathrm{I}_{\mathrm{DR}}+0,1 * \mathrm{I}_{\mathrm{CV}}
\end{aligned}
$$

Onde:

$\mathrm{I}_{\mathrm{AB}}=$ Indicador de Abastecimento de Água;

$\mathrm{I}_{\mathrm{ES}}=$ Indicador de Esgoto Sanitário;

$\mathrm{I}_{\mathrm{RS}}=$ Indicador de Resíduos Sólidos;

$\mathrm{I}_{\mathrm{DR}}=$ Indicador de Drenagem Urbana;

$\mathrm{I}_{\mathrm{CV}}=$ Indicador de Controle de Vetores.

Ainda, para a obtenção do valor desses indicadores secundários é necessária a realização de fórmulas específicas nas quais estão inseridos os indicadores de $3^{\mathrm{a}}$ ordem, como é mostrado na Tabela 1, onde também está relacionada a finalidade de cada indicador.

\begin{tabular}{|c|c|c|}
\hline INDICADORES DE $2^{\mathrm{a}}$ ORDEM & INDICADORES DE $3^{\mathrm{a}}$ ORDEM & FINALIDADE \\
\hline \multirow{3}{*}{$\begin{array}{l}\text { Indicador de Abastecimento } \\
\text { de Água }\left(\mathrm{I}_{\mathrm{AB}}\right)\end{array}$} & Indicador de cobertura de abastecimento $\left(\mathrm{I}_{\mathrm{CA}}\right)$ & $\begin{array}{l}\text { Quantificar os domicílios atendidos por sistemas de abastecimento } \\
\text { de água com controle sanitário }\end{array}$ \\
\hline & Indicador de qualidade da água distribuída $\left(\mathrm{I}_{\mathrm{OA}}\right)$ & Monitorar a qualidade da água fornecida \\
\hline & Indicador de saturação do sistema produtor $\left(\mathrm{I}_{\mathrm{SAP}}\right)$ & $\begin{array}{l}\text { Comparar a oferta e demanda para programar novos sistemas ou } \\
\text { ações que minimizem as perdas }\end{array}$ \\
\hline \multirow{2}{*}{$\begin{array}{l}\text { Indicador de Esgoto Sanitário } \\
\qquad\left(I_{E S}\right)\end{array}$} & Indicador de cobertura em coleta de esgoto $\left(\mathrm{I}_{\mathrm{CE}}\right)$ & Quantificar os domicílios atendidos por rede de esgoto \\
\hline & Indicador de esgoto tratado $\left(\mathrm{I}_{\mathrm{TE}}\right)$ & Qualificar os domicílios atendidos por rede de esgoto \\
\hline \multirow{4}{*}{$\begin{array}{l}\text { Indicador de Resíduos } \\
\text { Sólidos }\left(\mathrm{I}_{\mathrm{RS}}\right)\end{array}$} & Indicador de coleta de resíduos $\left(\mathrm{I}_{\mathrm{CR}}\right)$ & Quantificar os domicílios atendidos por coleta de resíduos \\
\hline & $\begin{array}{l}\text { Indicador de saturação do tratamento e } \\
\text { disposição final dos resíduos sólidos }\left(\mathrm{I}_{\mathrm{SR}}\right)\end{array}$ & Indicar a necessidade de novas instalações \\
\hline & Indicador de coleta seletiva $\left(\mathrm{I}_{\mathrm{CS}}\right)$ & Identificar em quais bairros há coleta seletiva e centro de triagem \\
\hline & $\begin{array}{l}\text { Indicador de acondicionamento de resíduos } \\
\text { sólidos }\left(\mathrm{I}_{\mathrm{AC}}\right)\end{array}$ & Identificar o acondicionamento dos resíduos em cada bairro \\
\hline \multirow{3}{*}{ Indicador de Drenagem $\left(I_{D R}\right)$} & Indicador de alagamento ou inundação $\left(\mathrm{I}_{\mathrm{Al}}\right)$ & $\begin{array}{l}\text { Identificar as vias com ou sem ocorrência de inundação ou } \\
\text { alagamento }\end{array}$ \\
\hline & Indicador de rua pavimentada $\left(I_{R P}\right)$ & Indicar vias com, parcialmente ou sem pavimentação \\
\hline & Indicador de uso do solo ( $\left.\mathrm{I}_{\mathrm{us}}\right)$ & $\begin{array}{l}\text { Identificar a contribuição do uso do solo em cada bairro para o } \\
\text { assoreamento do sistema de drenagem }\end{array}$ \\
\hline \multirow{3}{*}{$\begin{array}{l}\text { Indicador de Controle de } \\
\text { Vetores }\left(I_{\mathrm{Cv}}\right)\end{array}$} & Indicador de dengue $\left(\mathrm{I}_{\mathrm{VD}}\right)$ & Identificar a necessidade de programas preventivos \\
\hline & Indicador de esquistossomose $\left(\mathrm{I}_{\mathrm{VE}}\right)$ & $\begin{array}{l}\text { Identificar a necessidade de programas preventivos de redução e } \\
\text { eliminação de vetores transmissores e/ou hospedeiros da doença }\end{array}$ \\
\hline & Indicador de leptospirose $\left(\mathrm{I}_{\mathrm{VL}}\right)$ & $\begin{array}{l}\text { Identificar a necessidade de programas preventivos de redução e } \\
\text { eliminação de resíduos e ratos }\end{array}$ \\
\hline
\end{tabular}

Tabela 1: Indicadores de $2^{\mathrm{a}}$ e $3^{\mathrm{a}}$ ordem e suas finalidades.

Fonte: adaptado de Baggio (2013). 
De acordo com Batista e Silva (2006), o valor do ISA corresponde à soma de todos os indicadores, sendo que os valores podem variar de 0 a $100 \%$. E a interpretação dos valores se dá por meio da condição de salubridade ambiental do município, conforme a Tabela 2.

Tabela 2: Situação de salubridade por faixa de situação (\%).

\begin{tabular}{|c|c|}
\hline Situação de salubridade & Pontuação do ISA \\
\hline Insalubre & $0-25,50$ \\
\hline Baixa salubridade & $25,51-50,50$ \\
\hline Média salubridade & $50,51-75,50$ \\
\hline Salubre & $75,51-100,00$ \\
\hline \multicolumn{2}{|c|}{ Fonte: Batista e Silva (2006). }
\end{tabular}

Para a realização dos cálculos do ISA da área urbana do município de Frederico Westphalen foi utilizada a metodologia de Baggio (2013), de forma que o ISA/CS seja calculado pela média ponderada de indicadores secundários, por meio da Eq. 4.

$$
\begin{aligned}
& \text { ISA }=0,25 * I_{A B}+0,25 * I_{E S}+0,20 * I_{R S} \\
& +0,20 * I_{D R}+0,1 * I_{C V}
\end{aligned}
$$

Onde:

$\mathrm{I}_{\mathrm{AB}}=$ Indicador de Abastecimento de Água;

$\mathrm{I}_{\mathrm{ES}}=$ Indicador de Esgoto Sanitário;

$\mathrm{I}_{\mathrm{RS}}=$ Indicador de Resíduos Sólidos Urbanos;

$I_{D R}=$ Indicador de Drenagem Urbana;

$\mathrm{I}_{\mathrm{CV}}=$ Indicador de Controle de Vetores.

Para a obtenção do valor de cada um dos indicadores secundários foi necessária a realização de fórmulas específicas para Frederico Westphalen, nas quais inseridos os indicadores de $3^{\mathrm{a}}$ ordem. Dessa forma, foram realizadas adaptações para aplicação da metodologia do Indicador de Salubridade Ambiental de Cocal do Sul (ISA/CS) conforme a disponibilidade de dados e informações.

\subsection{Indicador de abastecimento de água $\left(\mathrm{I}_{\mathrm{AB}}\right)$}

$O$ indicador de $2^{\mathrm{a}}$ ordem $\mathrm{I}_{\mathrm{AB}}$ foi calculado a partir da média aritmética entre três indicadores de $3^{\mathrm{a}}$ ordem: o indicador de cobertura de abastecimento de água $\left(\mathrm{I}_{\mathrm{AC}}\right)$, o indicador de qualidade da água distribuída $\left(I_{Q A}\right)$ e o indicador de saturação do sistema produtor $\left(\mathrm{I}_{\mathrm{SAP}}\right)$, por meio da Eq. 5 .

$\mathrm{I}_{\mathrm{AB}}=\frac{\mathrm{I}_{\mathrm{AC}}+\mathrm{I}_{\mathrm{QA}}+\mathrm{I}_{\mathrm{SAP}}}{3}$

$\mathrm{O} \mathrm{I}_{\mathrm{CA}}$ foi calculado por meio da Eq. 6 e sua pontuação será de 0 a 100. 0 valor, quanto mais próximo de 100, indica uma melhor situação da cobertura de abastecimento de água.

$\mathrm{I}_{\mathrm{CA}}=\frac{\mathrm{D}_{\mathrm{UA}}}{\mathrm{D}_{\mathrm{UT}}} * 100(\%)$

Onde:

$\mathrm{D}_{\mathrm{UA}}=$ Domicílios urbanos atendidos por abastecimento de água;

$\mathrm{D}_{\mathrm{UT}}=$ Domicílios urbanos totais.

Os dados para a obtenção desse indicador foram conseguidos por meio da base de dados da Fundação de Economia e Estatística (FEE).

O cálculo do $\mathrm{I}_{\mathrm{QA}}$ foi realizado por meio da Eq. 7.

$\mathrm{I}_{\mathrm{QA}}=\mathrm{K} *\left(\frac{\mathrm{N}_{\mathrm{AA}}}{\mathrm{N}_{\mathrm{AR}}}\right) * 100$

Onde:

$\mathrm{K}=$ Número de amostras realizadas pelo número mínimo de amostras exigidas pela Portaria 2914/11, sendo que esse coeficiente deverá ser menor ou igual a 1. Portanto, se for maior que 1 , será considerado igual a 1 para fins de ajuste do cálculo. 
$\mathrm{N}_{\mathrm{AA}}=$ Quantidade de amostras consideradas como sendo de água potável relativa aos coliformes totais (mensais);

$\mathrm{N}_{\mathrm{AR}}=$ Quantidade de amostras realizadas (mensais).

A pontuação deste indicador de $3^{a}$ ordem se dá por meio da Tabela 3, a qual também demonstra a situação do mesmo.

Tabela 3: Pontuação e situação do indicador de $3^{a}$ ordem I QAA .

\begin{tabular}{|c|c|c|}
\hline $\mathrm{I}_{\mathrm{QA}}$ (\%) & Pontuação & Situação \\
\hline $\mathrm{I}_{\mathrm{OA}}=100 \%$ & 100 & Excelente \\
\hline $95<\mathrm{I}_{\mathrm{OA}}<99,9 \%$ & 80 & Ótima \\
\hline $85<\mathrm{I}_{\mathrm{QA}}<94,9 \%$ & 60 & Boa \\
\hline $70<\mathrm{I}_{\mathrm{OA}}<84,9 \%$ & 40 & Aceitável \\
\hline $50<\mathrm{I}_{\mathrm{OA}}<69,9 \%$ & 20 & Insatisfatória \\
\hline $\mathrm{I}_{\mathrm{OA}}<49,9 \%$ & 0 & Imprópria \\
\hline
\end{tabular}

Fonte: Adaptado de CONESAN (1999).

Os dados para calcular o $\mathrm{I}_{\mathrm{QA}}$ foram conseguidos por meio da Portaria 2.914/2011 e também junto à Companhia Riograndense de Saneamento (CORSAN). A CORSAN é a empresa responsável pelo abastecimento de água do município de Frederico Westphalen.

O Indicador de saturação do sistema produtor $\left(\mathrm{I}_{\mathrm{SAP}}\right)$ foi calculado por meio da Eq. 8, analisandose o tempo em que o sistema ficará saturado. Os dados referentes à esse indicador de $3^{\circ}$ ordem foram obtidos na unidade física da CORSAN.

$$
\mathrm{n}=\frac{\log \left(\frac{\mathrm{CP}}{\mathrm{VP} *\left(\frac{\mathrm{k} 2}{\mathrm{k} 1}\right)}\right)}{\log (1+\mathrm{t})}
$$

\section{Onde:}

$\mathrm{n}=$ Tempo em que o sistema ficará saturado (anos);

$\mathrm{CP}=$ Capacidade de produção (L/s);

$\mathrm{VP}=$ Volume de produção necessário para atender a população atual (L/s); $\mathrm{k} 1=$ Perda atual;

k2 = Perda prevista para 5 anos;

$t=$ Taxa de crescimento anual da população urbana para os 5 anos subsequentes ao ano base.

A Tabela 4 relaciona o valor encontrado de " $n$ " com a pontuação adequada do I_SAP.

Tabela 4: Pontuação do indicador de $3^{\mathrm{a}}$ ordem $\mathrm{I}_{\mathrm{SAP}}$.

\begin{tabular}{|c|c|c|c|}
\hline \multicolumn{2}{|c|}{ Valor de n } & Variação do $\mathbf{n}$ & Pontuação do $\mathrm{I}_{\text {SAP }}$ \\
\hline \multirow{2}{*}{0} & \multirow{2}{*}{5} & $0<\mathrm{n}<5$ & 100 \\
\hline & & $\mathrm{n} \leq 0$ & Interpolar \\
\hline
\end{tabular}

Fonte: Adaptado de CONESAN (1999).

\subsection{Indicador de esgoto sanitário (I_ES)}

Este indicador de $2^{\mathrm{a}}$ ordem foi calculado por meio da média aritmética entre dois indicadores de $3^{a}$ ordem, demonstrado na Eq. 9.

$\mathrm{I}_{\mathrm{ES}}=\frac{\mathrm{I}_{\mathrm{CE}}+\mathrm{I}_{\mathrm{TE}}}{2}$

Onde:

$\mathrm{I}_{\mathrm{CE}}=$ Indicador de cobertura em coleta de esgoto;

$\mathrm{I}_{\mathrm{TE}}=$ Indicador de esgotos tratados.

$O$ indicador de cobertura em coleta de esgoto $\left(\mathrm{I}_{\mathrm{CE}}\right)$ foi calculado por meio da Eq. 10.

$\mathrm{I}_{\mathrm{CE}}=\frac{\mathrm{D}_{\mathrm{UE}}}{\mathrm{D}_{\mathrm{UT}}} * 100$

Sendo:

$\mathrm{D}_{\mathrm{UE}}=$ Domicílios urbanos atendidos por coleta;

$\mathrm{D}_{\mathrm{UT}}=$ Domicílios urbanos totais.

A pontuação do $\mathrm{I}_{\mathrm{CE}}$ foi obtida por meio da relação apresentada na Tabela 5. 
Tabela 5: Pontuação do indicador $\mathrm{I}_{\mathrm{CE}}$.

\begin{tabular}{|c|c|}
\hline Variação do $\mathrm{I}_{\mathrm{CE}}$ & Pontuação do $\mathrm{I}_{\mathrm{CE}}$ \\
\hline $\mathrm{I}_{\mathrm{CE}}>90 \%$ & 100 \\
\hline $75<\mathrm{I}_{\mathrm{CE}}<89,9 \%$ & Interpolar \\
\hline $\mathrm{I}_{\mathrm{CE}}<75,9 \%$ & 0 \\
\hline
\end{tabular}

O Indicador de esgotos tratados (I_TE) foi calculado a partir da Eq. 11 e pontuado por meio da relação apresentada na Tabela 6.

$\mathrm{I}_{\mathrm{TE}}=\mathrm{I}_{\mathrm{CE}} * \frac{\mathrm{VT}}{\mathrm{VC}} * 100$

Onde:

$\mathrm{I}_{\mathrm{CE}}=$ Índice de esgotos coletados (\%);

$\mathrm{VC}=$ Volume coletado (número de domicílios atendidos * 4 hab/dom. * vazão diária de $160 \mathrm{~L} / \mathrm{s}$ ); $\mathrm{VT}=$ Volume tratado de esgoto $(=\mathrm{VC})$.

Tabela 6: Pontuação do indicador $\mathrm{I}_{\mathrm{TE}}$.

\begin{tabular}{|c|c|}
\hline Variação do $\mathrm{I}_{\mathrm{TE}}$ & Pontuação do $\mathrm{I}_{\mathrm{TE}}$ \\
\hline $\mathrm{I}_{\mathrm{TE}}>81 \%$ & 100 \\
\hline $45 \leq \mathrm{I}_{\mathrm{TE}} \leq 80,9 \%$ & Interpolar \\
\hline $\mathrm{I}_{\mathrm{TE}}<45,9 \%$ & 0 \\
\hline
\end{tabular}

Os dados para a realização de ambos os indicadores, tanto o $\mathrm{I}_{\mathrm{CE}}$ quanto o $\mathrm{I}_{\mathrm{TE}}$, foram obtidos na Secretaria Municipal do Meio Ambiente de Frederico Westphalen e através da base de dados da FEE.

\subsection{Indicador de resíduos sólidos urbanos $\left(I_{R S}\right)$}

O I_RS é um indicador de $2^{\mathrm{a}}$ ordem, cujo cálculo foi feito por meio da média aritmética dos valores dos indicadores de $3^{\mathrm{a}}$ ordem, podendo ser observado na Eq. 12.

$\mathrm{I}_{\mathrm{RS}}=\frac{\mathrm{I}_{\mathrm{CR}}+\mathrm{I}_{\mathrm{SR}}+\mathrm{I}_{\mathrm{CS}}+\mathrm{I}_{\mathrm{AC}}}{4}$
O valor do Indicador de Coleta de Resíduos $\left(I_{C R}\right)$ foi obtido por meio da Eq. 13.

$\mathrm{I}_{\mathrm{CR}}=\left(\frac{\mathrm{D}_{\mathrm{UC}}}{\mathrm{D}_{\mathrm{UT}}}\right) * 100$

Onde:

$\mathrm{D}_{\mathrm{Uc}}=$ Domicílios urbanos com coleta de resíduos; $D_{U T}=$ Domicílios urbanos totais.

A pontuação do $I_{C R}$ foi dada por meio da relação apresentada na Tabela 7.

Tabela 7: Pontuação do indicador $I_{C R}$.

\begin{tabular}{|c|c|}
\hline Variação do $I_{C R}$ & Pontuação do $I_{C R}$ \\
\hline$I_{C R} \geq 99 \%$ & 100 \\
\hline $95<I_{C R}<99 \%$ & Interpolar \\
\hline$I_{C R}<95 \%$ & 0 \\
\hline
\end{tabular}

Os dados para o cálculo do $\mathrm{I}_{\mathrm{CR}}$ foram obtidos por meio da base de dados da FEE e da Secretaria Municipal do Meio Ambiente de FW.

O Indicador de Saturação do tratamento e disposição final dos resíduos $\left(\mathrm{I}_{\mathrm{SR}}\right)$ foi calculado por meio da Eq. 14, analisando-se o valor de "n".

$\mathrm{n}=\frac{\log \left(\mathrm{CA} * \frac{\mathrm{t}}{\mathrm{VL}}\right)+1}{\log (1+\mathrm{t})}$

Onde:

$\mathrm{CA}=$ Capacidade restante do aterro (toneladas);

$\mathrm{VL}=$ Volume coletado de resíduos (média anual em tonelada);

$\mathrm{t}=$ Taxa de crescimento anual médio da população para os 5 anos subsequentes ao ano da elaboração do ISA (\%).

A pontuação deste indicador é dada por meio da relação expressa na Tabela 8: 
Tabela 8: Pontuação do indicador $\mathrm{I}_{\mathrm{SR}}$.

\begin{tabular}{|c|c|}
\hline Variação do $\mathbf{n}$ & Pontuação do $I_{\text {SR }}$ \\
\hline$n \geq 5$ & 100 \\
\hline $5>n>0$ & Interpolar \\
\hline$n \leq 0$ & 0 \\
\hline
\end{tabular}

A obtenção dos dados para este cálculo foi por meio do CIGRES (Consórcio Intermunicipal de Gestão de Resíduos Sólidos). O CIGRES é um consórcio formado por 30 municípios, localizado no município de Seberi - RS e foi criado como uma alternativa para atender à região de Frederico Westphalen no que diz respeitos a resíduos sólidos urbanos, viabilizando a implantação de novos programas e tecnologias que possam apoiar o desenvolvimento sustentável voltado para uma proteção do meio ambiente e para uma busca pela qualidade de vida da população.

Para pontuar o $\mathrm{I}_{\mathrm{CS}}$ foram observados os seguintes critérios:

a) Se há coleta seletiva e centro de triagem, a pontuação é 100;

b) Se há coleta seletiva, mas não há centro de triagem, a pontuação é 50;

c) Se não há coleta seletiva, mas há centro de triagem, a pontuação é 25;

d) Se não há coleta seletiva nem centro de triagem, a pontuação é 0 .

Para identificar esse indicador, as informações necessárias foram obtidas na Secretaria Municipal do Meio Ambiente de Frederico Westphalen.

O Indicador de Acondicionamento de Resíduos $\left(I_{A C}\right)$ é também um indicador de $3^{a}$ ordem, cuja pontuação foi dada observando-se os seguintes critérios:

a) Acondicionamento correto com lixeira padrão, a pontuação é 100; b) Acondicionamento correto com lixeira alternativa, a pontuação é 50;

c) Acondicionamento em sacolas (sem lixeira), a pontuação é 30;

d) Queima, enterro e outro destino dos resíduos, a pontuação é 0 .

Utilizaram-se dados primários para o levantamento desse indicador, sendo realizadas visitas a cada bairro da área urbana do município e observado o tipo de acondicionamento dos resíduos.

\subsection{Indicador de drenagem (I_DR)}

$O I_{D R}$ (indicador de $2^{a}$ ordem) foi calculado por meio da soma dos valores dos indicadores de $3^{a}$ ordem, conforme a Eq. 15.

$$
\mathrm{I}_{\mathrm{DR}}=\mathrm{I}_{\mathrm{AI}}+\mathrm{I}_{\mathrm{RP}}+\mathrm{I}_{\mathrm{US}}
$$

O Indicador de alagamento ou inundação $\left(I_{A}\right)$ é um indicador de $3^{\mathrm{a}}$ ordem, cujo cálculo é realizado por meio da Eq. 16, sendo posteriormente inserido diretamente na Eq. 15.

$\mathrm{I}_{\mathrm{AI}}=\mathrm{P} 1 *$ critério

Sendo P1 = 0,60 e o critério:

a) Com alagamento/inundação = 0; e

b) Sem alagamento/inundação $=1$.

O cálculo do Indicador de rua pavimentada $\left(I_{R P}\right)$ foi realizado por meio da Eq. 17 e inserido posteriormente na Eq. 15.

$\mathrm{I}_{\mathrm{RP}}=\mathrm{P} 2 *$ critério

Sendo P2 = 0,20 e o critério: 
a) Com pavimentação = 1;

b) Parcialmente pavimentado $=0,5 ; \mathrm{e}$

c) Sem pavimentação $=0$.

O Indicador de uso do solo $\left(\mathrm{I}_{\mathrm{us}}\right)$ tem como objetivo identificar a contribuição do uso do solo em cada bairro para o assoreamento do sistema de drenagem, sendo calculado por meio da Eq. $18 \mathrm{e}$ posteriormente inserido na Eq. 15.

$$
\mathrm{I}_{\mathrm{US}}=\mathrm{P} 3 * \text { critério }
$$

Sendo P3 = 0,20 e o critério:

a) Vegetação arbustiva - arbórea $=1$;

b) Pastagem =0,5;

c) Agricultura $=0,25 ; \mathrm{e}$

d) Sem vegetação $=0$.

Para classificar o resultado da drenagem utilizouse a metodologia de Batista e Silva (2006), de acordo com a Tabela 9.

Tabela 9: Classificação de drenagem.

\begin{tabular}{|c|c|}
\hline Intervalo de valores & Classificação \\
\hline$I_{D R} \geq 0,98$ & Excelente \\
\hline $0,98>\left.\right|_{D R} \geq 0,85$ & Muito boa \\
\hline $0,85>I_{D R} \geq 0,60$ & Boa \\
\hline $0,60>I_{D R} \geq 0,40$ & Regular \\
\hline $0,40>I_{D R} \geq 0,0$ & Ruim/Muito ruim \\
\hline Fonte: Adaptado de Batista e Silva (2006). \\
\hline
\end{tabular}

As informações necessárias para a obtenção dos indicadores de $3^{\mathrm{a}}$ ordem, $I_{A l}$ e $I_{R P}$, foram obtidas na Secretaria de Coordenação e Planejamento, na Prefeitura Municipal de Frederico Westphalen. Além disso, com o programa Google Earth Pro, foi possível obter os valores para a determinação do critério que indica o uso do solo (com vegetação, pastagem, agricultura ou sem vegetação), sendo feitos polígonos medindo a área de cada uso do solo, utilizando no cálculo do $I_{\text {US }}$ para observar o que possuiu a maior predominância de área.

\subsection{Indicador de controle de vetores (I $\left.{ }_{\mathrm{cV}}\right)$}

$\mathrm{O} \mathrm{I}_{\mathrm{CV}}$ foi calculado por meio da média ponderada de três indicadores de $3^{\mathrm{a}}$ ordem, conforme a Eq. 19.

$\mathrm{I}_{\mathrm{CV}}=\frac{\left[\left(\frac{\mathrm{I}_{\mathrm{VD}}+\mathrm{I}_{\mathrm{VE}}}{2}\right)+\mathrm{I}_{\mathrm{VL}}\right]}{2}$

O Indicador de Dengue $\left(\mathrm{I}_{\mathrm{VD}}\right)$, indicador de $3^{\mathrm{a}}$ ordem, foi observado no número de casos suspeitos. Então, se:

a) Bairro sem casos nos últimos 5 anos, a pontuação é de 100; e

b) Bairro com casos nos últimos 5 anos, a pontuação é de 0 .

$\mathrm{O}$ Indicador de Esquistossomose $\left(\mathrm{I}_{\mathrm{VE}}\right)$ é também um indicador de $3^{\mathrm{a}}$ ordem cuja identificação é feita por meio do número de casos. Então, se:

a) Bairro sem caso nos últimos 5 anos, a pontuação é de 100;

b) Bairro com incidência anual < 1, a pontuação é de 50;

c) Bairro com incidência 5 > inc. $\geq 1$, a pontuação é de 25; e

d) Bairro com incidência $\geq 5$, a pontuação é de 0 .

O Indicador de Leptospirose $\left(\mathrm{I}_{\mathrm{V}}\right)$, indicador de $3^{\mathrm{a}}$ ordem, foi identificado pelo número de casos. Então, se:

a) Bairro sem casos nos últimos 5 anos, a pontuação é de 100; e 
b) Bairro com casos nos últimos 5 anos, a pontuação é de 0 .

Para todos os indicadores de $3^{a}$ ordem referentes ao $\mathrm{I}_{\mathrm{CV}}$, o número de casos utilizado nos cálculos foi o de casos suspeitos de cada doença, em razão dos dados de casos confirmados não terem sido disponibilizados. Todas as informações necessárias para o cálculo do $\mathrm{I}_{\mathrm{cV}}$ foram obtidas na $19^{a}$ Coordenadoria Regional da Saúde no Setor de Vigilância Epidemiológica e Ambiental, localizada em Frederico Westphalen.

\section{RESULTADOS E DISCUSSÃO}

Para uma compreensão eficiente dos resultados deste trabalho, serão expostos a seguir os valores de cada indicador de $2^{\mathrm{a}}$ ordem com a discussão dos mesmos, e então, será apresentado e discutido o resultado final do indicador de $1^{\mathrm{a}}$ ordem (ISA/FW), a partir dos indicadores de $2^{\mathrm{a}}$ ordem.

\subsection{Indicadores de $2^{\text {a }}$ ordem}

\subsubsection{Indicador de Abastecimento de Água $\left(I_{A B}\right)$}

A partir dos indicadores de $3^{a}$ ordem $I_{C A}, I_{Q A}$ e $I_{S A P}$, obteve-se o resultado final do I_AB, como pode ser visto na Tabela 10.

Tabela 10: Resultado do Indicador de Abastecimento de Água $\left(\mathrm{I}_{\mathrm{AB}}\right)$.

\begin{tabular}{|c|c|c|c|}
\hline $\mathrm{I}_{\mathrm{CA}}$ & $\mathrm{I}_{\mathrm{OA}}$ & $\mathrm{I}_{\mathrm{SAP}}$ & $\mathrm{I}_{\mathrm{AB}}$ \\
\hline 95,57 & 100 & 100 & $\mathbf{9 8 , 5 3}$ \\
\hline
\end{tabular}

O valor obtido do $\mathrm{I}_{\mathrm{CA}}$ foi 95,57 devido ao fato de 7500 domicílios urbanos, do total de 7847, serem abastecidos pela rede geral de distribuição de água (CORSAN). O restante dos domicílios, 324 , é abastecido por poços ou nascentes e 23 são abastecidos por outras formas não especificadas, de acordo com os dados da FEE.

A pontuação obtida do $\mathrm{I}_{\mathrm{QA}}$ foi 100, indicando uma situação excelente, conforme a Tabela 3. Portanto, a qualidade da água distribuída está de acordo com a exigida pela Portaria 2.914/2011do Ministério da Saúde, a qual exige que sejam feitas, no mínimo, 16 amostras mensais em relação à coliformes totais. A CORSAN realiza 44 amostras mensais para todos os parâmetros exigidos (turbidez, $\mathrm{pH}$, cor, cloro residual, fluoretos, coliformes totais e $E$. coli), fazendo ainda mais amostras do que é exigido na Portaria 2.914/2011. Das 44 amostras realizadas, as mesmas 44 estão dentro do padrão.

Em relação ao $\mathrm{I}_{\mathrm{SAP}}$, sua pontuação foi de 100 , indicando que não há necessidade de programar novos sistemas de abastecimento de água nem ações para minimizar as perdas de água, sendo considerado satisfatório para os bairros da área urbana de FW.

O valor de 98,53 do $\mathrm{I}_{\mathrm{AB}}$ demonstra que as condições de abastecimento de água realizada pela CORSAN foram satisfatórias no ano de 2016 para a área urbana de FW.

\subsubsection{Indicador de Esgoto Sanitário (I $\left.{ }_{E S}\right)$}

Sendo $0 \mathrm{I}_{\mathrm{ES}} \mathrm{O}$ indicador que relata a situação do município em relação ao esgotamento sanitário, o mesmo foi calculado por meio dos indicadores de $3^{\mathrm{a}}$ ordem $\left(\mathrm{I}_{\mathrm{CE}}\right.$ e $\left.\mathrm{I}_{\mathrm{TE}}\right)$, chegando ao valor final do $\mathrm{I}_{\mathrm{ES}}$, demonstrado na Tabela 11.

Tabela 11: Resultado final do Indicador de Esgoto Sanitário $\left(\mathrm{I}_{\mathrm{ES}}\right)$.

\begin{tabular}{|c|c|c|}
\hline $\mathbf{I}_{\mathrm{CE}}$ & $\mathbf{I}_{\mathrm{TE}}$ & $\mathbf{I}_{\mathrm{ES}}$ \\
\hline 0 & 0 & $\mathbf{0}$ \\
\hline
\end{tabular}


Os valores dos indicadores de $3^{\mathrm{a}}$ ordem $\left(\mathrm{I}_{\mathrm{CE}}\right.$ e $\left.\mathrm{I}_{\mathrm{TE}}\right) \mathrm{e}$ do $I_{E S}$ foram 0 devido ao fato de que o município não possui rede coletora nem tratamento adequado de esgoto sanitário, consequentemente, indicando uma situação extremamente insatisfatória.

Segundo informações do secretário do meio ambiente de Frederico Westphalen, o município tem quatro estações de tratamento de efluente (ETEs) precárias devido à falta de manutenção (Fig. 1 e 2), duas delas com impossível acesso. As ETEs estão saturadas, já que não ocorre tratamento do esgoto que é levado de caminhão até as mesmas. Além disso, não é feita a retirada do lodo, tornando as ETEs incapazes de suportar a carga de efluente que recebem.

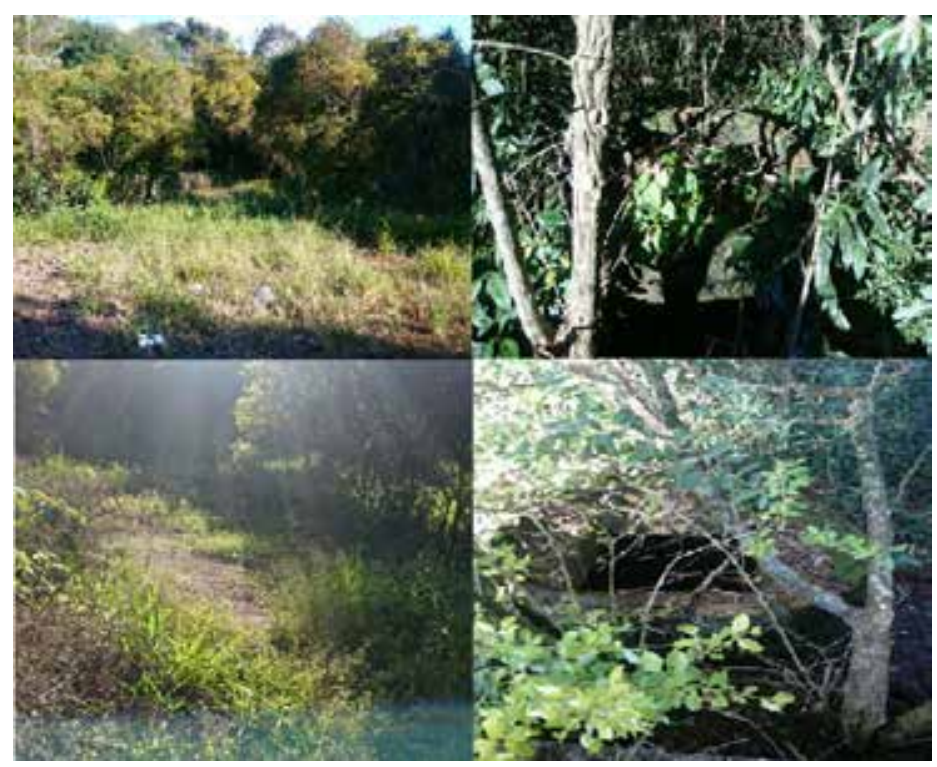

Figura 1: Estação de Tratamento de Efluente 1.

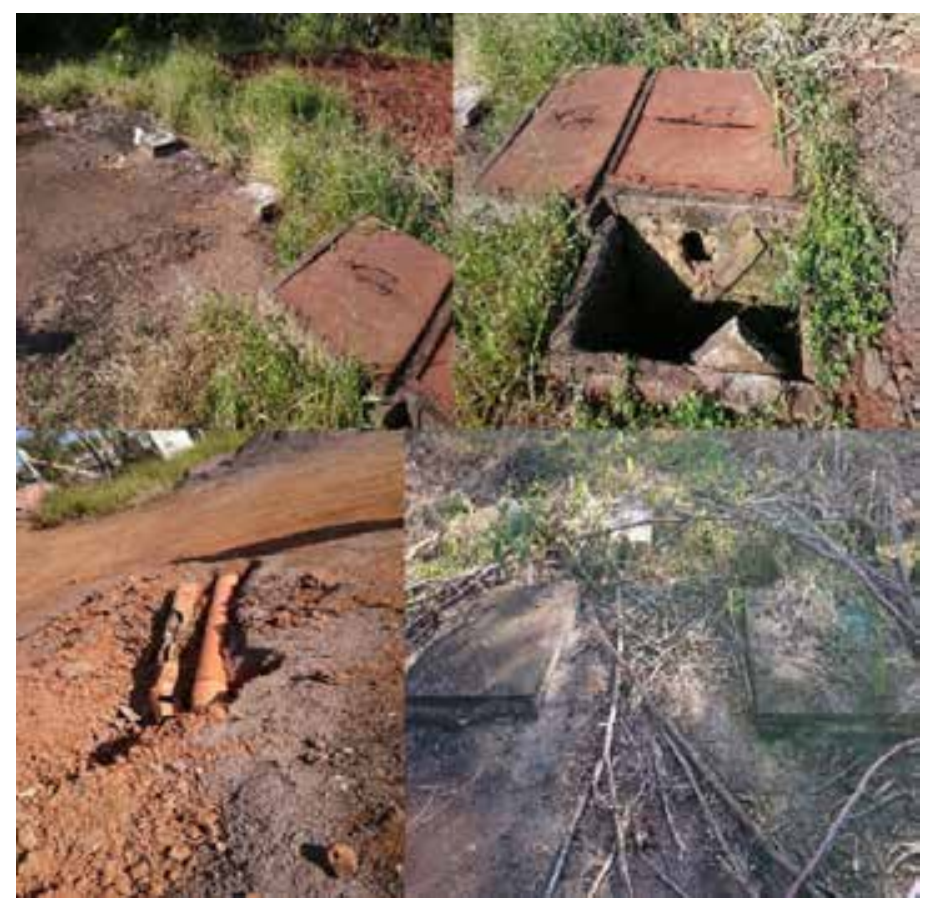

Figura 2: Estação de Tratamento de Efluente 2. 
A Secretaria Municipal do Meio Ambiente do município notificou a CORSAN para que fosse realizada a limpeza dessas estações, conseguindo-se um convênio no valor de $R \$ 212.000,00$ para a limpeza dessas duas estações.

A construção de novas redes coletoras de esgoto já foi iniciada e poderá atender $60 \%$ da população do município com capacidade de tratamento de $20 \mathrm{~L} / \mathrm{s}$. A conclusão desta obra tem previsão de 12 meses.

\subsubsection{Indicador de Resíduos Sólidos Urbanos (I $\left.{ }_{R S}\right)$}

O resultado final de cada indicador de $3^{\mathrm{a}}$ ordem referente ao I_RS está contido na Tabela 12 .

Tabela 12: Resultado final do Indicador de Resíduos Sólidos Urbanos $\left(\mathrm{I}_{\mathrm{RS}}\right)$.

\begin{tabular}{|c|c|c|c|c|}
\hline$I_{C R}$ & $I_{S R}$ & $I_{C S}$ & $I_{A C}$ & $I_{R S}$ \\
\hline 100 & 100 & 25 & 50 & $\mathbf{6 8 , 7 5}$ \\
\hline
\end{tabular}

A pontuação para o $I_{C R}$ foi de 100, pois todos os domicílios existentes possuem coleta de resíduos. A coleta de resíduos é realizada pelo $\mathrm{Cl}$ GRES e abrange todo o município, conforme informações do próprio consórcio.
Para o indicador de saturação do tratamento e disposição final dos resíduos sólidos $\left(\mathrm{I}_{\mathrm{SR}}\right)$, a pontuação foi de 100 para todos os bairros da área urbana do município, em função de haver tratamento adequado e disposição final dos resíduos sólidos, o qual é realizado pelo CIGRES, por meio de aterro sanitário e também de compostagem.

O I $\mathrm{I}_{\mathrm{CS}}$ obteve pontuação de 25 , devido ao fato de o município não possuir coleta seletiva, havendo apenas o centro de triagem no CIGRES, que realiza a triagem de todos os resíduos coletados na cidade. Segundo informações da Prefeitura Municipal de Frederico Westphalen, já foi realizada a tentativa de implantação do sistema de coleta seletiva, mas não houve disponibilidade de caminhões adequados para o serviço, além de outros fatores externos que interferiram, mas não foram informados.

O último indicador de $3^{\mathrm{a}}$ ordem que compõe o $\mathrm{I}_{\mathrm{RS}}$, o indicador de acondicionamento de resíduos sólidos $\left(l_{A C}\right)$, teve sua pontuação no valor de 50 . Esse valor, obtido por observação primária, se deu em razão de todos os bairros da área urbana do município possuírem um acondicionamento correto dos resíduos sólidos com coletoras alternativas (Fig. 3).

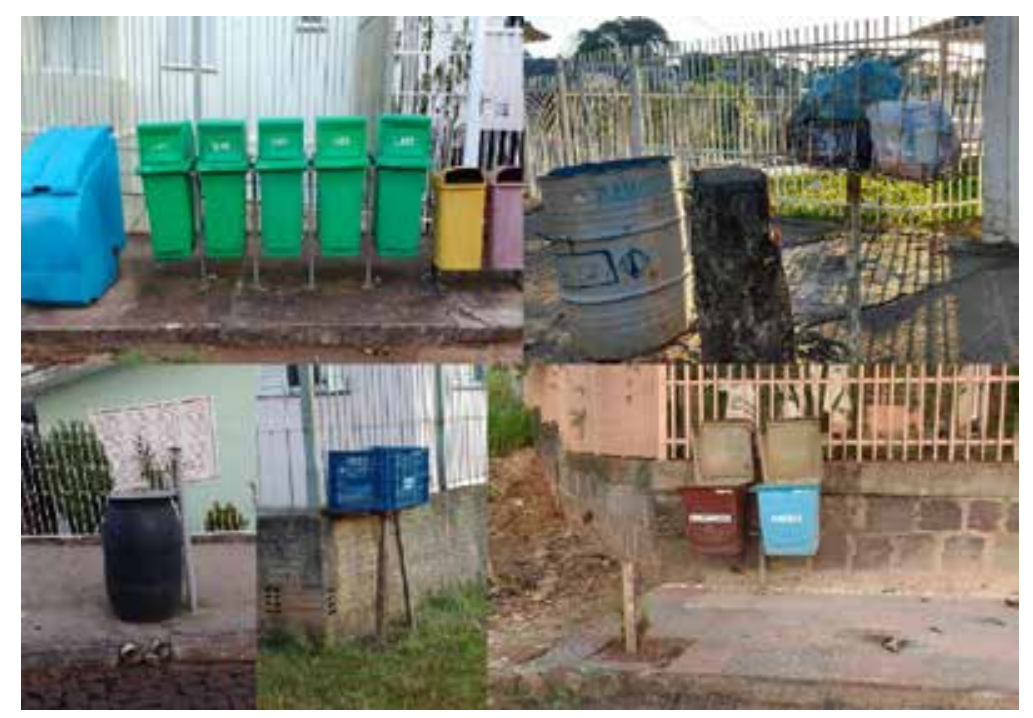

Figura 3: Acondicionamento dos resíduos sólidos com coletora alternativa. 
Nesta observação primária, pôde-se perceber que há pelo menos uma coletora alternativa em cada quadra do município, facilitando assim a deposição dos resíduos sólidos de uma forma correta por parte da população em geral.

O valor obtido para o indicador de resíduos sólidos $\left(I_{R S}\right)$ foi de 0,68. Tal valor demonstra que a situação do município em relação aos resíduos sólidos ainda deve ser melhorada. As coletoras devem ser do tipo padrão em todo o município, facilitando assim a separação dos resíduos. Além disso, o sistema de coleta seletiva deve ser implantado no município.

\subsubsection{Indicador de Drenagem Urbana (I ${ }_{D R}$ )}

O resultado final do indicador de $2^{\mathrm{a}}$ ordem $\mathrm{I}_{\mathrm{DR}}$ está inserido na Tabela 13, junto com os resultados de cada indicador de $3^{\mathrm{a}}$ ordem referente ao $\mathrm{I}_{\mathrm{DR}}$.

Tabela 13: Resultado do Indicador de Drenagem Urbana $\left(\mathrm{I}_{\mathrm{DR}}\right)$.

\begin{tabular}{|c|c|c|c|}
\hline $\mathbf{I}_{\mathrm{Al}}$ & $\mathbf{I}_{\mathrm{RP}}$ & $\mathbf{I}_{\mathrm{US}}$ & $\mathbf{I}_{\mathrm{DR}}$ \\
\hline 0,5 & 0,17 & 0,11 & $\mathbf{0 , 7 9}$ \\
\hline
\end{tabular}

Para o indicador de $3^{\mathrm{a}}$ ordem de alagamento ou inundação $\left(I_{A 1}\right)$, o valor médio obtido foi de 0,5 , indicando que em $16,66 \%$ dos bairros da área urbana de Frederico Westphalen há inundações ou alagamentos (Fig. 4).

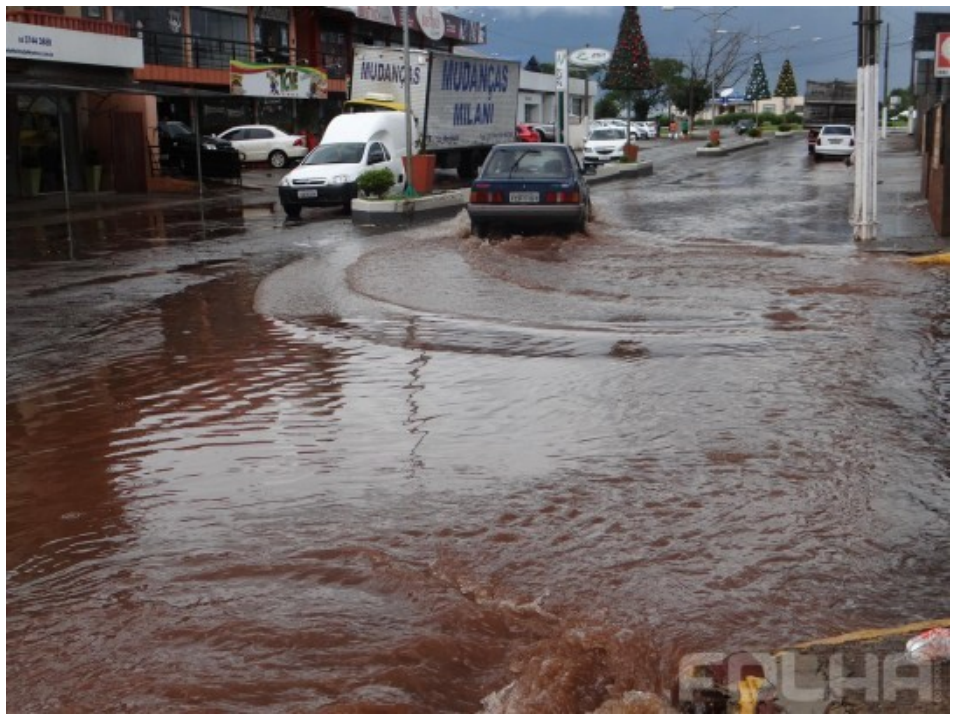

Figura 4: Alagamento na Rua Arthur Milani no Bairro Ipiranga em Frederico Westphalen. Fonte: Jornal Folha do Noroeste, 2013.

Segundo informações da Secretaria de Coordenação e Planejamento do município, estão sendo construídas galerias de drenagem pluvial, as quais possivelmente resolverão este problema.

0 valor médio obtido para o indicador de $3^{\mathrm{a}}$ ordem de rua pavimentada $\left(I_{R P}\right)$ foi de 0,17 . Esse valor indica que em 5,55\% dos bairros não há pavi- mentação, em 11,11\% há pavimentação parcial e em $83,33 \%$ dos bairros há pavimentação.

Para o indicador de uso do solo $\left(\mathrm{I}_{\mathrm{us}}\right)$, o valor obtido foi de 0,11 , demonstrando que em $44,44 \%$ dos bairros o uso do solo predominante do solo é de vegetação, em $27,77 \%$ dos bairros é de pastagem e em $27,77 \%$ não há vegetação. Para fins 
de cálculo, a área considerada sem vegetação foi aquela em que a maior parte da área total do bairro é predominantemente urbanizada.

De acordo com a Tabela 9, o valor do indicador de drenagem pode variar de 0 a 1 . Então, a partir do resultado obtido de 0,79, a área urbana de Frederico Westphalen foi considerada em uma condição boa.

\subsubsection{Indicador de Controle de Vetores $\left(I_{C V}\right)$}

A Tabela 14 apresenta o resultado final do indicador de $2^{\mathrm{a}}$ ordem $\mathrm{I}_{\mathrm{CV}}$ e também dos indicadores de $3^{\mathrm{a}}$ ordem necessários para obtenção do $\mathrm{I}_{\mathrm{CV}}$.

Tabela 14: Resultado do Indicador de Controle de Vetores $\left(\mathrm{I}_{\mathrm{CV}}\right)$.

\begin{tabular}{|c|c|c|c|}
\hline$I_{\mathrm{VD}}$ & $\mathrm{I}_{\mathrm{VE}}$ & $\mathrm{I}_{\mathrm{VL}}$ & $\mathrm{I}_{\mathrm{CV}}$ \\
\hline 38,88 & 100 & 66,66 & $\mathbf{6 8 , 0 5}$ \\
\hline
\end{tabular}

$O$ valor obtido para o indicador de $3^{\mathrm{a}}$ ordem referente à dengue $\left(\mathrm{I}_{\mathrm{VD}}\right)$ foi de 38,88 . Esse valor se deu devido ao fato de terem ocorridos inúmeros casos suspeitos da doença na área urbana do município nos últimos cinco anos. De acordo com informações do Setor de Vigilância Epidemiológica e Ambiental da 19a Coordenadoria Regional da Saúde, no período de janeiro a maio de 2016 a área urbana de Frederico Westphalen registrou em torno de 353 casos suspeitos de dengue, sendo o Bairro Fátima o local com mais incidência, com 126 casos suspeitos registrados.

Para o indicador de $3^{\mathrm{a}}$ ordem referente à esquistossomose, o valor obtido foi de 100, pois nos últimos cinco anos não foi registrado nenhum caso da doença, demonstrando assim que a água que circula na área urbana do município não possui vestígios do parasita da espécie Schistosoma, responsável pela propagação da doença.
0 indicador de $3^{\mathrm{a}}$ ordem que se refere à leptospirose obteve seu valor em 66,66. A razão deste valor foi devido à existência de registros de casos suspeitos da doença nos últimos cinco anos. Os bairros com registros da doença foram o Itapagé, Centro, Panosso, Santo Antônio, São Francisco de Paula e Jardim Primavera.

Dessa forma, a partir destes três indicadores de $3^{\mathrm{a}}$ ordem o valor médio obtido para o Indicador de Controle de Vetores (I_CV) foi de 68,05.

\subsection{Indicador de salubridade ambiental de Frederico Westphalen (ISA/FW)}

A partir dos resultados de cada indicador de $2^{\mathrm{a}}$ ordem exposto anteriormente, foi possível chegar ao resultado final do ISA/FW, o qual está inserido na Tabela 15, com cada valor obtido dos indicadores que compõem o ISA/FW.

Tabela 15: Resultado final do ISA/FW (\%).

\begin{tabular}{|c|c|c|c|c|c|}
\hline$I_{A B}$ & $I_{E S}$ & $I_{R S}$ & $I_{D R}$ & $I_{C V}$ & ISA/FW \\
\hline 98,52 & 0 & 68,75 & 79,44 & 68,05 & $\mathbf{6 1 , 0 7}$ \\
\hline
\end{tabular}

Foi obtido o valor de $61,07 \%$ como Indicador de Salubridade Ambiental para a área urbana do município de Frederico Westphalen, sendo caracterizado, de acordo com Silva (2006), como média salubridade.

A razão principal para o município ter sido considerado como média salubridade foi em função do Indicador de Esgoto Sanitário $\left(\mathrm{I}_{\mathrm{ES}}\right)$ ter seu valor resultado em zero, demonstrando, assim, a necessidade imediata de haver uma rede coletora de esgoto sanitário e um tratamento adequado do mesmo no município. A falta de investimento em uma área tão importante do saneamento básico torna o ambiente nocivo à saúde humana e também pode causar danos ao meio ambiente. 
Em relação aos outros indicadores de $2^{\mathrm{a}}$ ordem, os resultados demonstram uma situação satisfatória, porém ainda pode haver melhorias em cada um deles.

\subsection{Limitações do indicador de salubridade ambiental}

O ISA, em geral, apresentou algumas limitações na sua aplicação. A primeira limitação observada foi em relação ao peso atribuído a cada indicador, sendo que não há uma razão explícita para isso, podendo assim influenciar o valor final do ISA.

No Indicador de Abastecimento de Água $\left(I_{A B}\right)$, um dos itens analisados foi referente às amostras realizadas pela CORSAN, em relação à presença ou não de coliformes totais. No entanto, a $E$. coli é um indicador de extrema importância na qualidade da água, por se tratar de um patógeno transmissor de doenças de veiculação hídrica, o qual também deveria ser analisado.

O Indicador de Controle de Vetores $\left(I_{C V}\right)$ deveria considerar o número de casos, nos últimos cinco anos, de doenças diarreicas, as quais estão relacionadas à mortalidade infantil.

Ainda, o ISA aparenta deficiências no modo de estruturar e ponderar o amplo e diversificado conjunto de indicadores de primeira, segunda e terceira ordem e deixa a desejar quando comparadas diferentes realidades municipais. Dessa forma, as variáveis consideradas deveriam ser exploradas em um aspecto mais amplo e, quando poderadas em soma, deveriam se arranjar em um indicador plausível, interpretando de forma eficiente diferentes realidades municipais.

\section{CONCLUSÃO}

O indicador de esgoto sanitário influenciou de maneira significativa a salubridade ambiental da área urbana do município, por não haver coleta nem mesmo tratamento do esgoto sanitário, mostrando a necessidade da implantação da rede coletora de esgoto e de uma estação de tratamento de efluente.

Por outro lado, o indicador de abastecimento de água para a área urbana de FW indica que a população tem bom acesso a esse serviço, pois 95,57\% dos domicílios são abastecidos por rede geral de distribuição de água.

Em relação ao indicador de resíduos sólidos, foi demonstrado que, com mudanças básicas, como a implantação da coleta seletiva dos resíduos sólidos, distribuição de coletoras padrões e atividades de educação ambiental voltadas para a população, esse indicador pode chegar a uma condição excelente.

Algumas melhorias precisam ser efetuadas também com relação ao indicador de drenagem, devendo-se investir em sistemas de drenagem pluvial que consigam evitar alagamentos e inundações em algumas áreas dos bairros que apresentaram esses problemas para, assim, chegarse à uma condição totalmente satisfatória.

$\mathrm{O}$ indicador de controle de vetores apresentou uma boa pontuação, entretanto essa pontuação pode ser aumentada por meio da realização dos investimentos sugeridos nas áreas de coleta e tratamento de esgoto e, também, de drenagem urbana.

Dessa forma, a área urbana de FW foi considerada como sendo de média salubridade, demostrando que o município ainda carece de alguns serviços necessários para ser considerado salubre. Com a aplicação do ISA/FW na área urbana do município de Frederico Westphalen, notou-se que esse indicador é um instrumento muito útil para o planejamento do saneamento ambiental e priorização dos investimentos públicos, visto 
que engloba uma série de indicadores relativos ao tema.

Além disso, recomenda-se que se façam atualizações periódicas para indicadores como o ISA/ FW, pois a aplicação de indicadores visa representar um momento definido. Consequentemente, quanto mais o município investir em políticas públicas ambientais, mais as condições de saneamento serão melhoradas e o ISA/FW tenderá a aumentar a sua pontuação.

Outro aspecto a ser destacado é a inexistência de Indicadores de Salubridade Ambiental no estado do Rio Grande do Sul, pois o valor do ISA/FW é um valor isolado ou pontual. Para uma maior eficácia da metodologia, comparações entre municípios serviriam de auxílio adicional para o diagnóstico integrado de salubridade ambiental.

\section{CONTRIBUIÇÃO DOS AUTORES}

Todos os autores contribuíram de forma igualitária.

\section{REFERÊNCIAS}

BAGGIO, D. B. Aplicação do Indicador de Salubridade Ambiental (ISA) no município de Cocal do Sul -SC. 2013.132 p. Trabalho de Conclusão de Curso (Graduação em Engenharia Ambiental). Universidade do Extremo Sul Catarinense, Criciúma, 2013.

BATISTA, M. E. M.; SILVA, T. C. da. 0 modelo ISA/JP - Indicador de performance para diagnóstico do saneamento ambiental urbano. 2006. 10 f. UFPB, Paraíba, 2005.
CONSELHO ESTADUAL DE SANEAMENTO (CONESAN). ISA - Indicador de Salubridade Ambiental: Manual Básico. São Paulo: Companhia de Saneamento Básico do Estado de São Paulo, 1999.

FUNDAÇÃO DE ECONOMIA E ESTATÍSTICA (FEE). Feedados. 2016. Disponível em: <http://www.fee.rs.gov.br/> Acesso em: 20 mai. 2018.

GAZZEH, K.; ABUBAKAR, I. R. Regional disparity in access to basic public services in Saudi Arabia: A sustainability challenge. Utilities Policy, Saudi Arabia, v. 52, n. 1, p. 70-80, jun. 2018.

HELLER, L. Relação entre saúde e saneamento na perspectiva do desenvolvimento. Ciência \& Saúde Coletiva, v. 3, n. 2, p. 73-84, 1998.

HUTTON, G.; CHASE, C. Water supply, sanitation and hygiene. $3^{\mathrm{a}}$ ed. Washington, DC: Injury Prevention and Environmental Health, 2017. 27 p.

JORNAL FOLHA DO NOROESTE. Chuva causa alagamentos em Frederico Westphalen. 2016 Disponível em: <http://www.folhadonoroeste.com.br/site/noticia/101-chuva-causa-alagamentos-em-frederico-westphalen>. Acesso em: 10 jun. 2018.

MONTGOMERY, M. et al. No end to cholera without basic water, sanitation and hygiene. Bulletin Of The World Health Organization, Switzerland, v. 96, n. 6, p.371-372, jun. 2018.

PERARD, E. Economic and financial aspects of the sanitation challenge: A practitioner approach. Utilities Policy, Luxembourg, v. 52, n. 1, p. 22-26, jun. 2018.

SAIANI, C. C. S.; TONETO JÚNIOR, R. Evolução do acesso a serviços de saneamento básico no Brasil (1970 a 2004). Economia e Sociedade, Brasil, v. 19, n. 1, p.79-106, abr. 2010.

SILVA, N. V. S. As condições de salubridade ambiental das comunidades periurbanas da bacia do baixo gramame: diagnóstico e proposição de benefícios. Dissertação UFPB/CT - João Pessoa, 2006.

TAKALA, A. Understanding sustainable development in Finnish water supply and sanitation services. International Journal of Sustainable Built Environment, Finland, v. 6, n. 2, p. 501-512, dez. 2017. 